\title{
Online Optimal Power Control of an Offshore Oil-Platform Power System
}

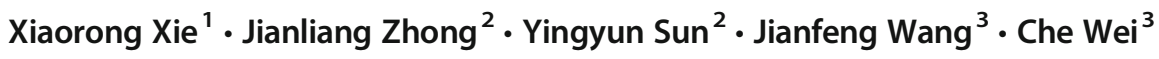 \\ Received: 2 August 2015 / Accepted: 7 November 2018 / Published online: 16 November 2018 \\ (C) Springer Nature Singapore Pte Ltd. 2018
}

\begin{abstract}
Offshore oil-platform power systems are important infrastructure for the exploitation of maritime oil and gas. However, its current energy management system, with relatively simple control scheme and low-level automation, can hardly operate the system in a secure and economic manner to match the rapid progress of offshore oil and gas exploitation. To address this issue, an online multi-objective optimal power control strategy is proposed and implemented based on the existing SCADA system. By incorporating network loss, gas consumption and voltage deviation into a synthesized objective function and taking the various operational constraints into account, the power-control task is formulated into a mixed-integer nonlinear optimized problem. And then an efficient procedure that combines the interior point method and the fast branch and bound method is developed to solve the problem. Consequently the optimal control strategy can be obtained online and either system security or operational efficiency is achieved. The developed control system has been put into practical use in the Weixinan offshore oil-platform power system (China's first of its kind). Field test results show that it can coordinate active and reactive power for online optimal control as per the changes of operating conditions. The improved safety, efficiency and power quality of the power system will definitely promote exploitation of offshore oil and gas in the respect of both security and efficiency.
\end{abstract}

Keywords Offshore oil-platform power system · Optimal power control $\cdot$ Mixed-integer nonlinear problem $\cdot$ Interior point method $\cdot$ Fast branch and bound method

Xiaorong Xie

xiexr@tsinghua.edu.cn

Jianliang Zhong

zhongj1@ncepu.edu.cn

Yingyun Sun

sunyy@ncepu.edu.cn

Jianfeng Wang

wangjf@cnooc.com.cn

Che Wei

weiche@cnooc.com.cn

1 State Key Laborarory of Power System, Department of Electrical Engineering, Tsinghua University, Haidian District, Beijing 100084, China

2 State Key Laboratory of Alternate Electrical Power System with Renewable Energy Source, North China Electric Power University, Changping District, Beijing 102206, China

3 China National Offshore Oil Corporation Research Institute, Chaoyang District, Beijing 100033, China

\section{Introduction}

Offshore oil resource is playing an increasing role in satisfying our fossil fuel needs. It accounts for over one third of the world's total oil production. For China in 2013, $19 \%$ of crude oil is from shallow offshore reserves [1] and the proportion continues to rise at very high speed [2]. In the offshore oil industry, the power supply system is one of the most important infrastructures to ensure safe and efficient production. How to reliably power offshore oil platforms is becoming a critical issue. However, previously and currently, most oil platforms far from the land are powered by standalone power stations built on them. This power supply mode, though simple, is criticized for high investment and low reliability. Moreover, there is a great risk of blackout at the platform once the power station thereon shuts down. Therefore, to improve the reliability of power supply, it becomes a trend to develop offshore oilplatform power system that electrically connect multiple platforms to supply power in a manner of network [3]. In 2010, the first interconnected offshore power system, called the Weixinan offshore oil-filed power system (WOOPS), was established on the South China Sea (SCS) around the 
Weizhou Island. Since then, more and more offshore platforms have been connected electrically along the coast of China and many regional interconnected offshore power systems have been developed or under construction. Therefore, how to efficiently operate and control those offshore power systems for reliable power supply is critical for the effective and safe operation of offshore oil platforms.

For the offshore oil-platform power systems, there are emergency control system and quasi-steady-state control system dealing with the dynamic stability problem and efficient energy management issue, respectively. This paper mainly focuses on the latter, intending to offer optimal power control strategy for such an interconnected offshore oil-platform power system. Through global optimization of generation, var. compensation and transformer tap options, the reliability and quality of power supply can be improved and the cost of generation is reduced while the load demands be met in a safe and stable manner. Compared with the inland power system and its energy management and control system, which is mature through many years' development $[4,5]$, the offshore power system has unique system features and control requirements:

1) The control infrastructure is relatively weak. Most of the systems only have the basic function of supervisory control and data acquisition (SCADA) and are short of model support and advanced control functions. It is urgent to improve automation and intelligence of the control system.

2) The primary power sources are gas turbine generators and the system is characterized by small inertia, high response speed and closely coupling of active and reactive power. Coordinated control of both active and reactive power is required to reconcile power balance, voltage quality and economic operation within a short period (e.g. $0.5 \mathrm{~s}$ ) so as to achieve online quasi-real-time decision-making. This is quite different from the inland power system, in which the online control of AGC and AVC is difficult to coordinate and the time of decision-making is relatively long (say minute-level) due to the huge scale and numerous variables of the power system $[6,7]$.

3) Gas turbine generators have very rapid response, but it is prone to be tripped in case of slight overload due to its limited overloading capability. As for the submarine cables, the charging power is very high and the overloading capacity is limited by the thermal stability. The investment of gas turbines and submarine cables is extremely high. So it is required that the power system operate within the tolerance to guarantee equipment safety and service life.

4) The oilfield associated gas, which is used as the fuel of power generation, has very high economic value. In addition, to comply with the strict claim of emission reduction and environment protection on the sea, the power generation cost and energy losses should be minimized.

5) Decision variables of power control include continuous variable (such as unit / STATCOM output) and discrete variable (such as on/off status of shunt reactors and transformer tap position). So the optimization problem is generally a mixed-integer nonlinear one. It is a big challenge to find the solution efficiently on line to achieve quasireal-time control. But this difficulty is usually avoided for the inland power system through multi-step separate decision.

Therefore, the power control method widely used in inland power systems is almost inappropriate for the offshore system. A special power control strategy and system is in urgent need. However, very few research was done in this aspect. References $[8,9]$ investigated the impact of wind power on the operation of offshore oil-platform power systems, however, without optimal control of the overall system. In [10], security and stability control system for offshore power grids was analyzed, however, not touching the topic of operating the power system optimally.

Based on the requirements and status quo of the power control technology of offshore oil-platform power systems, a multi-objective optimal power control system is proposed and implemented for a practical system in this paper. The innovative contributions of this work includes:

1) The developed power control system is of a serviceoriented architecture (SOA) [11] and based on common information model (CIM). So all its basic functions such as topology analysis, state estimation and advanced control applications such as online power optimization are plug-and-play.

2) The optimal power control task is formulated into a constrained, mixed-integer nonlinear programming problem. The goal is to synthetically minimize power generation cost, network loss rate and bus voltage deviation. Moreover, the gas consumption of generators can be updated online based on operation data.

3) An efficient algorithm combining the interior point method (IPM) and the fast branch-and-bound method (BBM) is designed to solve the optimization problem and thus rapid decision-making and online power control is achieved.

4) The online optimal power control system is put into operation in China's first offshore oil-platform power system, i.e., WOOPS. Field test results verify its practicability and effectiveness.

The rest of the paper is organized as follows: In "The Offshore Oil-Platform Power System and its Improved 
Power Control System" section, an improved configuration of the power control system is proposed based on the analyses of the status quo of WOOPS and its existing control strategy. "Formulation of the Optimal Power Control Problem" section details the formulation of the power control problem. In "Solution to the Optimization Problem" section, IPM and fast $\mathrm{BBM}$ are combined to solve the optimization problem efficiently. In "Implementation of OPC and its Application Results" section, the developed control system is applied to the target system to investigate its actual performance. Finally, brief conclusions are drawn in the sixth section.

\section{The Offshore Oil-Platform Power System and its Improved Power Control System}

\section{Description of the Target Offshore Oil-Platform Power System}

The target system, or WOOPS, is located to the southwest of Weizhou Island, Beibu Gulf, South China Sea. It is China's first large-scale and multi-unit oil-platform power system. Figure 1 shows its geographic diagram. The system consists of six offshore drilling platforms and one processing terminal on the Weizhou Island (namely WZIT). Among them, WZ111, WZIT and WZ12-1PAP are platforms with gas turbine generators while the other three platforms are only of load. All platforms are connected via submarine cables and thus form an interconnected offshore oil-platform power system. The total load is in the range of $10 \mathrm{MW}$ to $18 \mathrm{MW}$. The system consists of 11 gas turbine generators, six at WZIT, three at WZ12-1PAP and two at WZ11-1. The rated capacity of each generator varies from 2.5 MW to 4.5 MW. Under normal situations, nine generators are in service and the other two in cold standby. This power system not only provides necessary power for oil and gas production but also supplies electricity to residents on the island.

Figure 2 is the single-line diagram of the system. The figure marks out the platform name and the basic parameters of the system, including rated bus voltage, per-unit impendence of transformer and submarine cables with the base capacity of 10MVA. Active/reactive power ranges of generators and a Static Synchronous Compensator (STATCOM, which is mounted at WZ11-1 N) are listed in Table 1. The transformer connecting WZ11-1 and WZ11-4 $\mathrm{N}$ has a fixed tap ratio of $10.5 / 6.3 \mathrm{kV}$. Other transformers are of on-load tap changers (OLTC), with ratio range being $35 \pm 4 \times 2.5 \% / 6.3 \mathrm{kV}$.

\section{The Originally SCADA System and Power Control Strategy}

In order to monitor and control the power system, a simple energy management system (EMS) is originally deployed. It only has basic SCADA functions and power control strategy. The SCADA collects operation data of the power system by the way of polling. The data includes switching status, RMS voltage and current, active and reactive power, etc. They are acquired from the measuring points of system at fixed

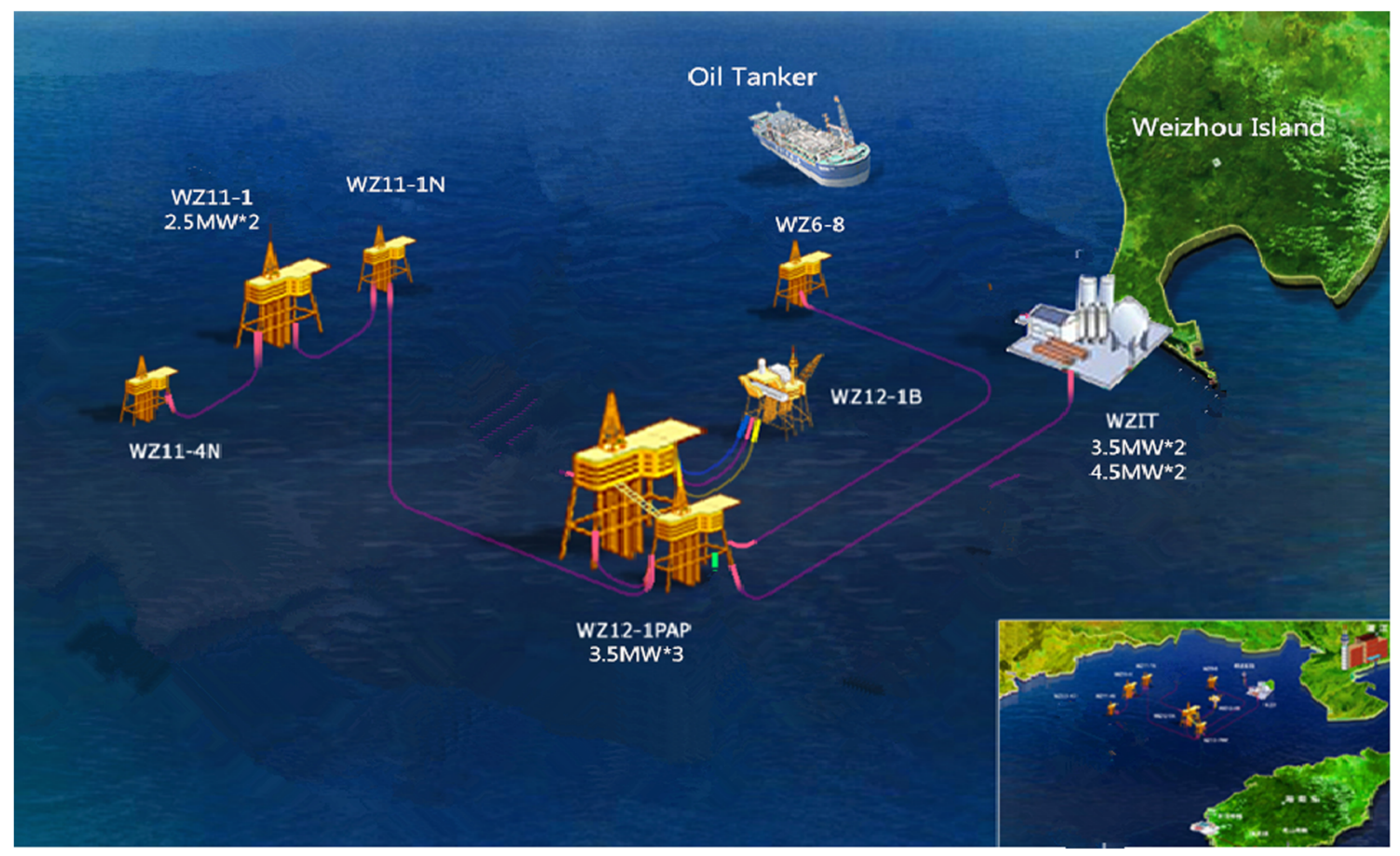

Fig. 1 Geographic diagram of Weixinan offshore oil-platform power system 


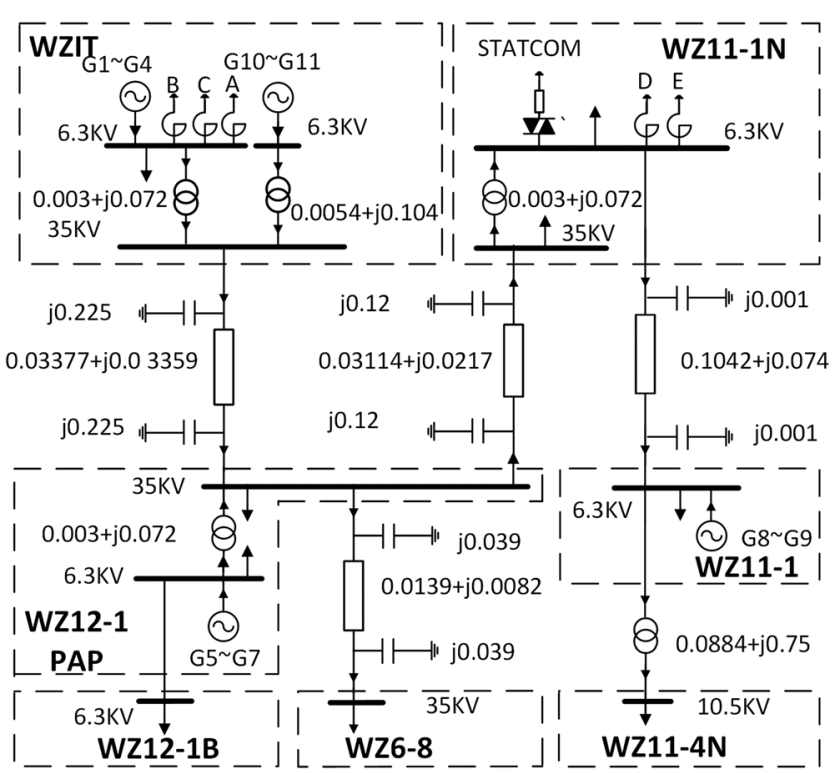

Fig. 2 Single-line diagram of Weixinan offshore oil-platform power system

intervals. Then the data and status are directly displayed on the single-line diagram for the users to monitor the current conditions of the system.

Control of active power is carried out following a simple strategy: EMS/SCADA gathers the MW output of generators and load at all buses. Then the loss of network is worked out. All online generators share the total load plus network loss with a same MW loading level. In other words, the active power output of each generator is determined by

$P_{\mathrm{G} i}=k_{\mathrm{L}} P_{G i}^{\max }$

$k_{\mathrm{L}}=\left(P_{\mathrm{L}}+P_{\mathrm{Loss}}\right) / \sum_{i=1}^{N_{\mathrm{G}}} P_{\mathrm{G} i}^{\max }$

where $P_{G i}$ and $P \max G i$ are active output (p.u.) and maximum active output (p.u.) of generator $i$ respectively; $N_{G}$ is total number of generators; $k_{L}$ is the unified load rate of the system; $P_{L}$ is the active power load of the system; $P_{\text {Loss }}$ is the loss of the network.

The reactive power of each generator is regulated with either of the three methods: i) Maintaining the terminal voltage, which is achieved by the automatic voltage regulation

Table 1 Active/reactive power ranges of generators and STATCOM

\begin{tabular}{lll}
\hline Generator/STATCOM & Active power(MW) & Reactive power(Mvar) \\
\hline G1 G7 & $0 \sim 3.5$ & $-1 \sim 2.5$ \\
G8 G9 & $0 \sim 2.5$ & $-0.5 \sim 1.8$ \\
G10 G11 & $0 \sim 4.5$ & $0 \sim 3.5$ \\
STATCOM & 0 & $-3.0 \sim 3.0$ \\
\hline
\end{tabular}

(AVR) of the excitation system by tracking a pre-defined voltage reference. ii) Dispatching the reactive power, which is fulfilled by EMS through sending reactive power order for each generator. 3) Keeping the power factor constant. For a generator, its reactive power control mode can be switched among these 3 ones as required by the operator at different operating conditions or time period.

The existing EMS/SCADA system with the abovementioned power control strategy, though simple and easy for implementation, has obvious flaws:

1) SCADA just displays the acquired data to the users. The acquisition and transmission of data is asynchronously conducted and inevitably introduces errors. Lack of basic functions such as topology analysis and state estimation tend to make inconsistent and inaccurate presentation of the system. As a result, accurate system state information is not available and the resultant operation may be deviated or even incorrect, which definitely endangers the safety and stability of the whole power system.

2) The power control strategy is derived from the original single-platform power supply system, without considering the various constraints in a power grid, for instance, stability of the whole system, the capacity limit of cables and transformers. So under unfavorable conditions, such a simple control strategy would cause overload of equipment or even weaken system stability.

3) Economy and efficiency of system operation are not fully considered. With the existing control strategy, the active power of each generator only depends on the total load and its capacity. No consideration is given to the difference in generation cost and network loss caused by the transmission of power. When the distribution of loads is not matched with the capacity of deployed generator(s) on each platform or the operation cost of generator varies greatly, the existing control strategy would cause high fuel cost, increased network loss, and thereby degraded operation efficiency.

4) Relatively low-level automation and intelligence cannot comply with the growing expansion trend of offshore oil-platform power systems. Currently, there are huge number of non-automatic operations in the existing system, for instance, setting the var. reference of generators/STATCOM, switching transformer tap positions and reactors. The reliance on human experience and manual operations, on the one hand, seriously lowers the automation of the power system, and on the other hand, certainly threaten the security and reliability of the system. This is especially the case when the scale of the offshore oil platforms increases steadily. 


\section{Configuration of the Improved Power Control System}

In order to address the above issues, we develop an SOAbased power control system, of which the structure is shown in Fig. 3. The basic operation process is as follows: The SCADA acquires data from each platform of the offshore power system and integrate them into the common information model (CIM) [12,13], which is later used for the functions of topology analysis [14] and state estimation [15-17]. In this way, accurate operating information is obtained, which can be displayed on the graphical user interface (GUI) for the operators and also used as basic data of the optimal power control (OPC) module. Meanwhile, the OPC module receives control parameters from GUI and then executes optimal decision process automatically and periodically to get the optimal value of control variables. The control information is next transmitted through communication networks to actuators on platforms, which finally execute the control commands to adjust generator/STATCOM output, change transformer tap options, switch on/off shunt reactors and etc. The above operations are executed repeatedly until the system reaches the optimal operation condition. The operators can supervise the whole optimal control process with the GUI at any time. Since the control system is developed based on SOA, each functional module is a plug and play service. As the key function of the entire control system, OPC will be the focus of discussion in the following part of this paper.

\section{Formulation of the Optimal Power Control Problem}

With the requirements and limitations of the offshore oilplatform power system fully taken into account, the optimal power control task is mathematically formulated into a mixed-

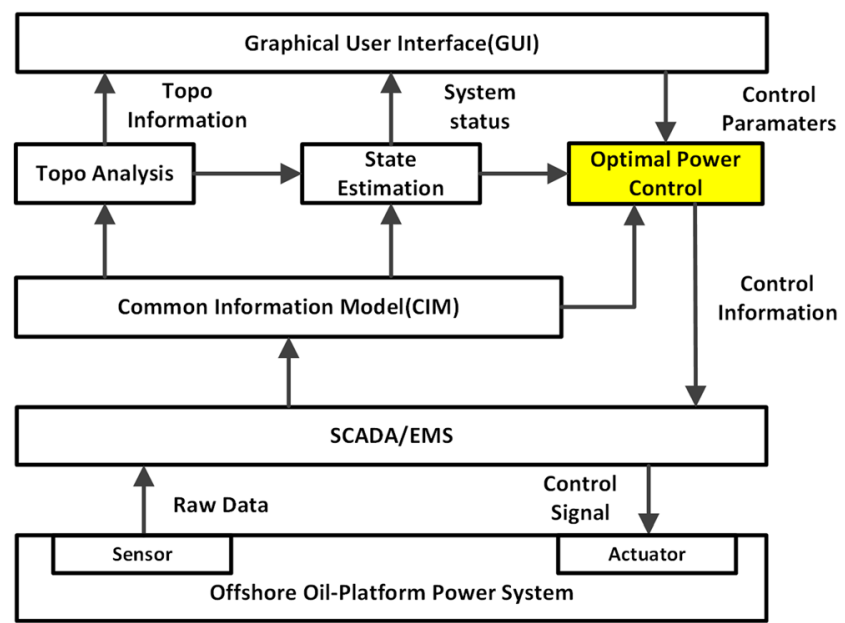

$f_{\mathrm{V}}=\sum_{i=1}^{N}\left(V_{\mathrm{x} i}^{2}+V_{\mathrm{y} i}^{2}-V_{\mathrm{n}}^{2}\right)^{2}$

where $V_{x i}, V_{y i}$ are real and imaginary parts of bus voltage; $V_{n}$ is the nominal voltage; $N$ is number of buses.

\section{Constraints}

i) Power flow constraint: For each bus or node, the active/ reactive power should be balanced, i.e.,

$\left\{\begin{array}{c}P_{i}-V_{\mathrm{x} i} \sum_{j=1}^{N}\left(G_{i j} V_{\mathrm{x} j}-B_{i j} V_{\mathrm{y} j}\right)-V_{\mathrm{y} i} \sum_{j=1}^{N}\left(G_{i j} V_{\mathrm{y} j}+B_{i j} V_{\mathrm{x} j}\right)=0 i=1, \ldots, N \\ Q_{i}-V_{\mathrm{y} j} \sum_{j=1}^{N}\left(G_{i j} V_{\mathrm{x} j}-B_{i j} V_{\mathrm{y} j}\right)+V_{\mathrm{x} i} \sum_{j=1}^{N}\left(G_{i j} V_{\mathrm{y} j}+B_{i j} V_{\mathrm{x} j}\right)=0 i=1, \ldots, N\end{array}\right.$

Fig. 3 Configuration of the improved power control system 
where $P_{i}, Q_{i}$ are net injected active and reactive power at busi, which is worked out by subtracting load from generator output at that bus; $G_{i j}$ and $B_{i j}$ are transfer conductance and susceptance between buses $i$ and $j$.

ii) Generator power constraint: The active/reactive power of each generator should be within its allowed range, or:

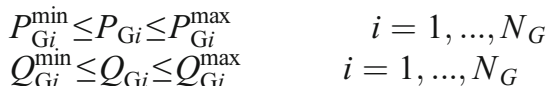

where $Q_{G i}$ is reactive output; $P \min G i$ is the minimum active output; $Q \min G i$ and $Q \max G i$ are minimum and maximum reactive output; the subscript $i$ denotes the $i$ th generator.

iii) Constraint on the capacity of cables: The power transmitted through a cable is limited by its physical condition:

$\left\{\begin{array}{l}P_{i j}=V_{\mathrm{x} i}\left(G_{i j} V_{\mathrm{x} j}-B_{i j} V_{\mathrm{y} j}\right)+V_{\mathrm{y} i}\left(G_{i j} V_{\mathrm{y} j}+B_{i j} V_{\mathrm{x} j}\right)-G_{i j}\left(V_{\mathrm{x} i}^{2}+V_{\mathrm{y} i}^{2}\right) \\ Q_{i j}=V_{\mathrm{y} i}\left(G_{i j} V_{\mathrm{x} j}-B_{i j} V_{\mathrm{y} j}\right)-V_{\mathrm{x} i}\left(G_{i j} V_{\mathrm{y} j}+B_{i j} V_{\mathrm{x} j}\right)+B_{i j}\left(V_{\mathrm{x} i}^{2}+V_{\mathrm{y} i}^{2}\right)\end{array}\right.$

$\left(P_{i j}^{2}+Q_{i j}^{2}\right)-\left(S_{i j}^{\max }\right)^{2} \leq 0$

where $Q_{G i}, Q_{G i}$ are active and reactive power flow from bus $i$ to $j$; Smax $i j$ is the maximum transfer capacity of the cable between bus $i$ and $j$.

iv) Bus voltage constraint: The magnitude of bus voltage should be within its upper and lower limits, namely

$\left(V_{i}^{\min }\right)^{2} \leq V_{\mathrm{x} i}^{2}+V_{\mathrm{y} i}^{2} \leq\left(V_{i}^{\max }\right)^{2} \quad i=1, \ldots, N$

where Vmin $i$ and Vmax $i$ are the upper and lower limits of voltage at busi.

v) Constraint on STATCOM var. output: STATCOM's var. output should be within its capacity range, or

$Q_{\mathrm{S} i}^{\min } \leq Q_{\mathrm{S} i} \leq Q_{\mathrm{S} i}^{\max } i=1, \ldots, N_{\mathrm{ST}}$

where $Q \min S i$ and $Q \max S i$ are minimum and maximum var. output; $N_{S T}$ is the number of STATCOMs.

vi) Constraint on tap positions of OLTC transformers: There are only a finite number of tap positions, or

$$
\begin{aligned}
k_{i}=k_{i 0} & +z_{i} \times \Delta_{i} \% \\
& \\
i & =1, \ldots, N_{\mathrm{T}}, \quad-n_{i} \leq z_{i} \leq n_{i} \quad z_{i} \in Z
\end{aligned}
$$

where $k_{i 0}$ and $k_{i}$ are nominal and actual ratios; $z_{i}$ is the tap position; $\Delta_{i} \%$ is the step of tap positions; $n_{i}$ is total number of tap positions.

vii) Reactor state constraint: There are two states for each reactor, namely 0 for off and 1 for on, or

$S T_{i} \in\{0,1\} \quad i=1, \ldots, N_{\mathrm{SR}}$

where $S_{T i}$ denotes the on/off state of a shunt reactor; $N_{S R}$ is the number of shunt reactors.

3. The integrated mathematical model

Based on the above objective function and constraints, the power control function of an offshore oil-platform power system can be formulated into the following constrained mixedinteger nonlinear optimization problem.

$$
\begin{aligned}
& \min f=\omega_{\mathrm{C}} f_{\mathrm{C}}+\omega_{\mathrm{P}} f_{\mathrm{P}}+\omega_{\mathrm{V}} f_{\mathrm{V}}
\end{aligned}
$$

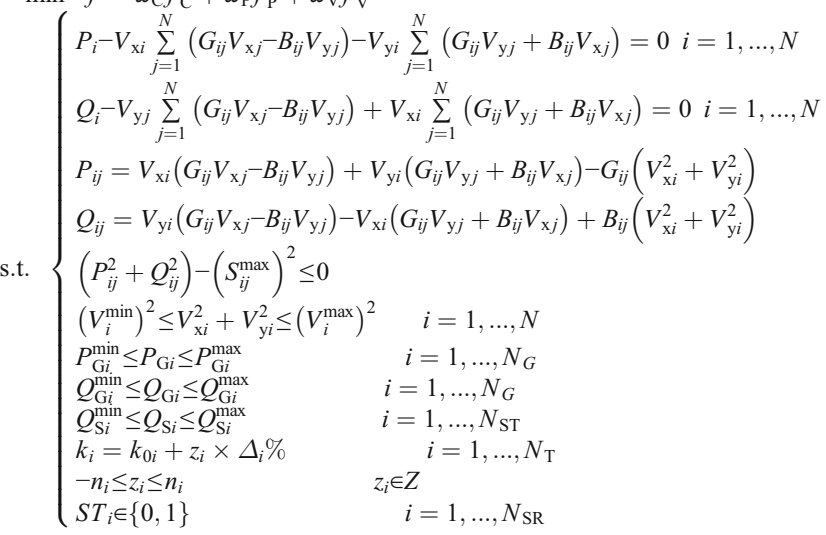

\section{Solution to the Optimization Problem}

The optimal power control problem (15) involves continuous variables (e.g., generator power and STATCOM var. output) and discrete variables (e.g., tap positions and on/off states of shunt reactors). It can be re-written in the following general form.

$\min f\left(\mathbf{x}_{\mathrm{c}}, \mathbf{x}_{\mathrm{d}}\right)$

s.t. $\left\{\begin{array}{l}\mathbf{h}\left(\mathbf{x}_{\mathrm{c}}, \mathrm{x}_{\mathrm{d}}\right)=0 \\ \mathrm{~g}^{\min } \leq \mathrm{g}\left(\mathbf{x}_{\mathrm{c}}, \mathrm{x}_{\mathrm{d}}\right) \leq \mathrm{g}^{\max } \\ \mathbf{x}_{\mathrm{c}} \in\left\{\mathbf{x}_{\mathrm{c}} \mid \mathbf{x}_{\mathrm{c}} \in R, \mathrm{x}_{\mathrm{c}} \mathbf{m i n}_{\mathrm{c}} \leq \mathrm{x}_{\mathrm{c}}^{\max }\right\} \\ \mathrm{x}_{\mathrm{d}} \in\left\{\mathrm{x}_{\mathrm{d}} \mid \mathrm{x}_{\mathrm{d}} \in Z, \mathrm{x}_{\mathrm{d}}^{\min } \leq \mathrm{x}_{\mathrm{d}} \leq \mathrm{x}_{\mathrm{d}}^{\max }\right\}\end{array}\right.$

where $f$ is the objective function; $\mathbf{x}_{\mathbf{c}}, \mathbf{x}_{\mathbf{d}}$ are vectors of continuous and discrete variables; $\mathbf{x m a x} \mathbf{c} / \mathbf{x m i n} \mathbf{c}$ and xmax $\mathbf{d} / \mathbf{x m i n} \mathbf{d}$ denote their upper/lower limits; $\mathbf{h}$ represents the equality functions; $g$ represent the inequality 
functions and $\mathbf{g}^{\mathbf{m a x}} / \mathbf{g}^{\mathbf{m i n}}$ are their upper and lower bounds, respectively.

The control-design problems (15) and (16) are both mixedinteger nonlinear optimization problems. It is hard for conventional methods to find a proper solution directly. Current solutions to such a problem generally include deterministic algorithms [18] and heuristic algorithms [19]. Heuristic algorithms, for instance, the particle swarm optimization method $[20,21]$ and genetic algorithm [22, 23], can handle discrete variables well. However, due to its relatively lower speed of converging and uncertainty in reaching optimal solution, the heuristic algorithm can hardly meet the need of online optimal power control. So in this paper the deterministic algorithm is adopted to solve the control problem. In previous literature [24], the interior point method (IPM) and the branch-andbound method (BBM) were combined to find the solution of similar problems. Its basic procedure is as follows: relax discrete variables first and solve the relaxed problem by IPM; then if any discrete variable in said solution does not meet its discrete constraint, break the original problem into several branch ones by BBM; next, branch problems are solved to update the upper and lower limits of the objective function; by repeating the former steps, the boundary of decisive variables will be narrowed gradually and the feasible optimal solution can be obtained in the end. In theory, this combined method can be used to solve the optimization problem (16). In practice, however, it has an obvious drawback: with the growth of the scale of the problem, the branches will increase dramatically, which in turn leads to very slow computation speed and poor converging performance. As a result, online execution of the optimal power control can hardly be achieved.

As far as WOOPS is concerned, it has eight discrete decision variables, i.e., five reactors and three OLTC transformers. Taking the nine tap positions of each OLTC transformer into account, there are totally $2^{5} \times 9^{3}$ branches to be traversed in the worst case if the conventional BBM is used [25]. Actually this is a huge number, which will definitely reduce the calculation speed and increase the possibility of non-convergence, which, consequently leads to the failure of the online power control function. To address this issue, we improve the traditional BBM into a fast one, which is then combined with the primal-dual IPM to solve our control problem efficiently. The general procedure, as shown in Fig. 4, is carried out in the following steps:

Step 1: Input parameters necessary for establishing the optimization model, including the parameters of power networks, the upper/lower operational limits of generators, transformers, submarine cables, the online updated parameters of gas consumption curve for each generator, and the weights in the objective function.
Step 2: Formulate the optimal power control task into the mathematical problem as shown in (15), which is later transformed into the general form of (16).

Step 3: Set the initial values for all decision variables. Although the primal-dual IPM is insensitive to initial conditions, the results of current state estimation are assigned as the initial values so that the convergence speed can be greatly accelerated.

Step 4: Relax the optimization problem. The IPM requires all variables be continuous while the power optimization model (16) has many a discrete variables (for instance, transformer tap positions, reactor on/off states). So the process of relaxing is necessary for the use of IPM.

Firstly, by relaxing the discrete variables (i.e., regarding them as continuous variables) and the inequality constraints (i.e, introducing the barrier parameter), the original problem can be transformed into the following form:

$$
\begin{aligned}
& \min f(\mathbf{x})-\mu \sum \log \left(\mathrm{s}_{1}\right)-\mu \sum \log \left(\mathrm{s}_{2}\right) \\
& \text { s.t. }\left\{\begin{array}{l}
\mathbf{h}(\mathbf{x})=\mathbf{0} \\
\mathbf{g}(\mathbf{x})+\mathbf{s}_{1}=\mathbf{g}^{\mathbf{m a x}}, \mathbf{g}(\mathbf{x})-\mathbf{s}_{2}=\mathbf{g}^{\text {min }} \\
\mathbf{x} \in\left\{\mathbf{x} \mid \mathbf{x} \in R, \mathbf{x}_{\min } \leq \mathbf{x} \leq \mathbf{x}_{\max }\right\} \\
\mathbf{s}_{1}, \mathbf{s}_{2} \in R^{+}
\end{array}\right.
\end{aligned}
$$

where $\mathbf{x}=\left[\mathbf{x}_{\mathbf{c}} ; \mathbf{x}_{\mathbf{d}}\right] ; \mathbf{s}_{\mathbf{1}}$ and $\mathbf{s}_{\mathbf{2}}$ are newly introduced relaxing variables; $\mu$ is the barrier parameter, the detail of which can be found in [26].

Then, by using the Lagrange multiplier, Eq.(17) is further converted into the following Lagrangian function:

$$
\begin{aligned}
L & =f(\mathbf{x})-\lambda^{T} \mathbf{h}(\mathbf{x})-\mathbf{z}^{T}\left[\mathbf{g}(\mathbf{x})-\mathbf{s}_{2}-\mathbf{g}^{\min }\right] \\
& -\mathbf{w}^{T}\left[\mathbf{g}(\mathbf{x})+\mathbf{s}_{1}-\mathbf{g}^{\max }\right]-\mu \sum \log \left(\mathbf{s}_{1}\right)-\mu \sum \log \left(\mathbf{s}_{2}\right)
\end{aligned}
$$

where $\lambda$ (any $\lambda \neq 0, \lambda \in \lambda$ ), $\mathbf{z}($ any $z>0, z \in \mathbf{z})$ and $\mathbf{w}($ any $w>0$, $w \in \mathbf{w})$ are Lagrange multipliers of the equality/inequality constraints, which are also known as the dual variables.

So the relaxed optimization problem can be obtained as

$$
\begin{aligned}
& \min L\left(\mathbf{x}, \lambda, \mathbf{z}, \mathbf{w}, \mu, \mathbf{s}_{1}, \mathbf{s}_{2}\right) \\
& \text { s.t. }\left\{\begin{array}{l}
\forall \lambda \neq 0, \lambda \in \lambda \\
\forall z>0, \quad z \in \mathbf{z} \\
\forall w<0, w \in \mathbf{W} \\
\mathbf{x} \in\left\{\mathbf{x} \mid \mathbf{x} \in R, \mathbf{x}^{\text {min }} \leq \mathbf{x} \leq \mathbf{x}^{\max }\right\}
\end{array}\right.
\end{aligned}
$$

Step 5: Solve the relaxed problem (19) with IPM. We have developed an IPM solver based on the open source package IPOPT (Interior Point Optimizer) [27] of COIN-OR community to effectively solve the optimization problem. It is fulfilled with the 4 sub-steps, of which the flow chart is also shown in Fig. 5. 


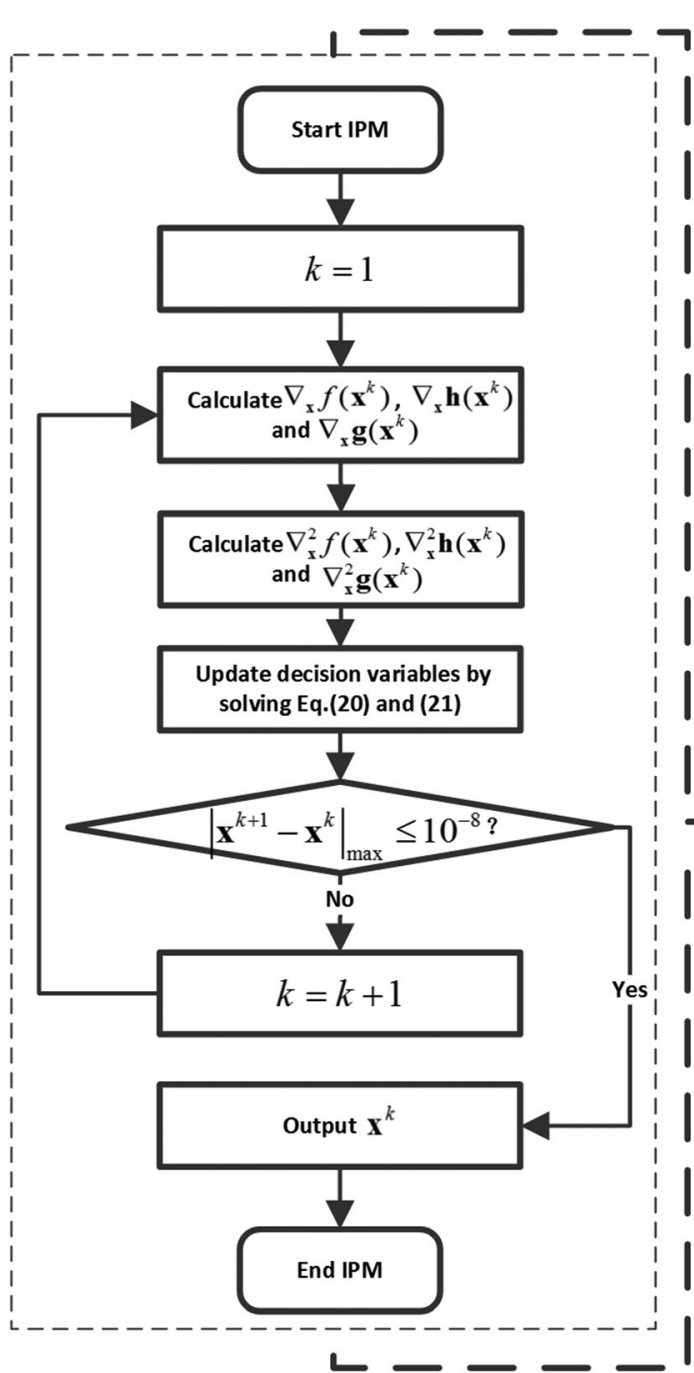

Fig. 4 Flow chart of the combined method of IPM and BBM

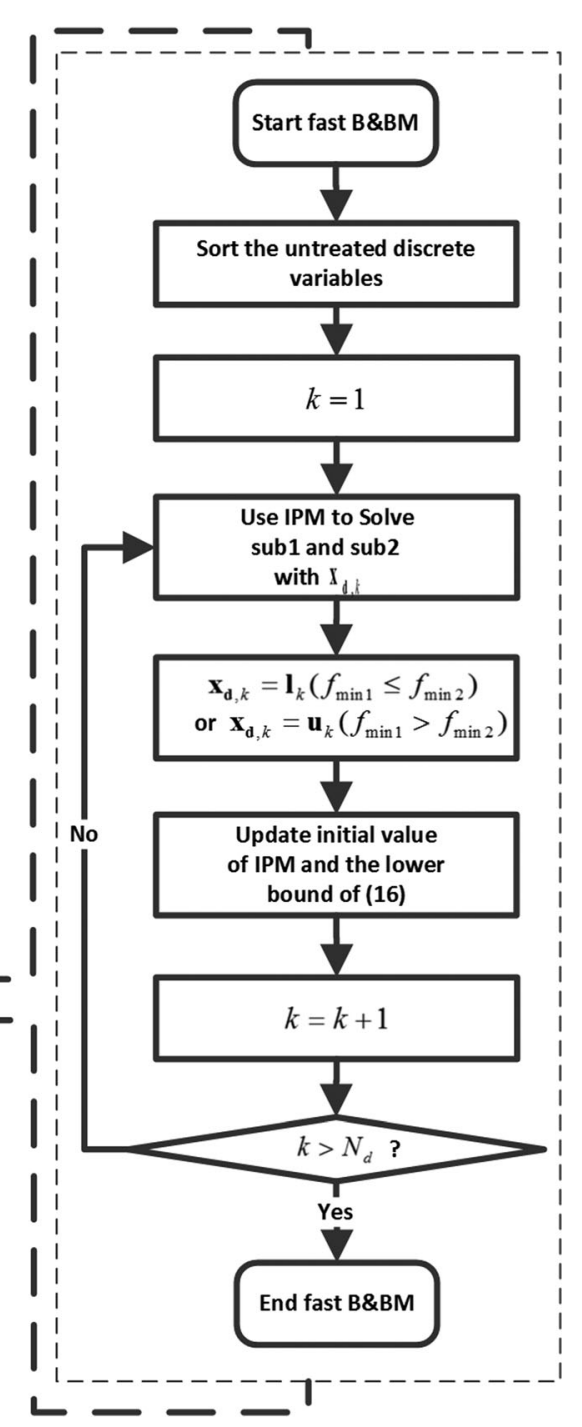

Substep 5-1: Calculate the first-order derivative of the objective function $\left(\nabla_{\mathbf{x}} f(\mathbf{x})\right)$ and the Jacobian matrices of the constraint functions $\left(\nabla_{\mathbf{x}} \mathbf{h}(\mathbf{x}), \nabla_{\mathbf{x}} \mathbf{g}(\mathbf{x})\right)$.

Substep 5-2: Calculate the second-order derivative of the objective function $(\nabla 2 \times f(\mathbf{x}))$ and the Hessian matrices of constraint functions $(\nabla 2 \mathbf{x h}(\mathbf{x}), \nabla 2$ $\mathbf{x g}(\mathbf{x}))$ by using the quasi-Newton approximation [27].

Substep 5-3: Update the decision variables. First, the Newton direction [28] is obtained by solving Eq. (20):

$\left[\begin{array}{cccccc}\mathbf{H} & \nabla_{\mathrm{x}} \mathbf{h}(\mathbf{x}) & \nabla_{\mathrm{x}} \mathbf{g}(\mathbf{x}) & \nabla_{\mathrm{x}} \mathbf{g}(\mathbf{x}) & 0 & 0 \\ \nabla_{\mathrm{x}}^{T} \mathbf{h}(\mathbf{x}) & 0 & 0 & 0 & 0 & 0 \\ \nabla_{\mathrm{x}}^{T} \mathbf{g}(\mathbf{x}) & 0 & 0 & 0 & -I & 0 \\ \nabla_{\mathrm{x}}^{T} \mathbf{g}(\mathbf{x}) & 0 & 0 & 0 & 0 & I \\ 0 & 0 & \mathbf{S}_{2} & 0 & \mathbf{Z} & 0 \\ 0 & 0 & 0 & \mathbf{S}_{1} & 0 & \mathbf{W}\end{array}\right]\left[\begin{array}{c}\Delta \mathbf{x} \\ \Delta \mathbf{\lambda} \\ \Delta \mathbf{z} \\ \Delta \mathbf{w} \\ \Delta \mathbf{s}_{2} \\ \Delta \mathbf{s}_{1}\end{array}\right]=\left[\begin{array}{c}L_{\mathrm{x}} \\ L_{\lambda} \\ L_{\mathrm{z}} \\ L_{\mathrm{w}} \\ L_{\mathrm{s}_{2}} \\ L_{\mathrm{s}_{1}}\end{array}\right]$

where $\mathrm{H}=-[\nabla 2 \times \mathrm{x} f(\mathbf{x})-\mu \nabla 2 \mathrm{xh}(\mathbf{x})-\nabla 2 \times \mathbf{x g}(\mathbf{x})(\mathbf{z}+\mathbf{w})], L_{x}, L_{\lambda}, L_{z}$, $L_{w}, L_{s 1}, L_{s 2}$ are the first-order derivatives of the Lagrangian function for each of the variables, $\mathbf{S}_{\mathbf{1}}=\operatorname{diag}\left(s_{1,1}, s_{1,2}, \ldots s_{1, \mathrm{r}}\right)$, $\mathbf{S}_{\mathbf{2}}=\operatorname{diag}\left(s_{2,1}, s_{2,2}, \ldots s_{2, \mathrm{r}}\right), \mathbf{W}=\operatorname{diag}\left(w_{1}, w_{2}, \ldots w_{\mathrm{r}}\right), \mathbf{Z}=\operatorname{diag}(-$ $\left.z_{1}, z_{2}, \ldots z_{\mathrm{r}}\right), r$ is the number of inequality constraints and $I$ is unit matrix.

Then the decision variables can be update by the following formulas [26]:

$$
\begin{aligned}
& \mathbf{x}^{k+1}=\mathbf{x}^{k}+\alpha_{\mathrm{p}} \Delta \mathbf{x}, \mathbf{s}_{1}^{k+1}=\mathbf{s}_{1}^{k}+\boldsymbol{\alpha}_{\mathrm{p}} \Delta \mathbf{s}_{1} \\
& \mathbf{s}_{2}^{k+1}=\mathbf{s}_{2}^{k}+\alpha_{\mathrm{p}} \Delta \mathbf{s}_{2}, \lambda^{k+1}=\lambda^{k}+\boldsymbol{\alpha}_{\mathrm{d}} \Delta \lambda \\
& \mathbf{w}^{k+1}=\mathbf{w}^{k}+\boldsymbol{\alpha}_{\mathrm{d}} \Delta \mathbf{w}
\end{aligned}
$$

where $\alpha_{\mathbf{p}}$ and $\alpha_{\mathbf{d}}$ are the vectors of primal-step length and the dual-step length, respectively.

Substep 5-4: Judge the convergence of IPM: If the criterion, or $\left|\mathbf{x}^{\mathrm{k}+1}-\mathbf{x}^{\mathrm{k}}\right| \leq 10^{-8}$, is met, the iteration converges and the optimal solution to the relaxed 


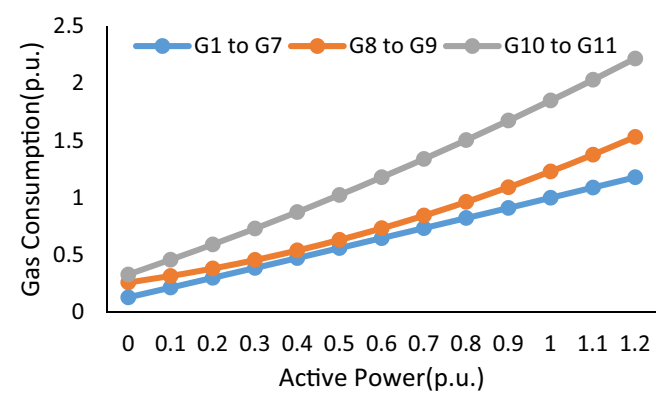

Fig. 5 Gas consumption curves of gas turbines

problem (19) is obtained, which are denoted as $\left(\mathbf{x}^{*} \mathbf{c}, \mathbf{x}^{*} \mathbf{d}\right)$. Otherwise, go back to Substep 5-1 and proceed to next iteration, where the last updated variables will be used as the new initial values.

Step 6: Discretize the solution. If any of the discrete variables is not assigned with its allowed value (later called: untreated), the fast BBM will be called to discretize that variable. Unlike the conventional BBM, which, in the worst case, has to traverse each branch, the fast BBM used here only considers the smaller branch of the objective function. Its basic procedure, as illustrated in Fig. 4, is carried out by the following sub-steps.
Substep 6-1: Sort the untreated discrete variables of the optimal solution obtained in the previous step. Since the tap positions of OLTC transformers have greater impact on system voltage and reactive power flow, they will be discretized first. Moreover, priority should be given to the taps of higher voltage levels. For the transformers at the same voltage level, their tap variables should be treated according to the order from that with the largest distance to its discrete value. Reactors go next. Similarly, they are treated in the descending order of their deviation from the discrete values. Suppose the sorted discrete variables are $\mathbf{x}_{\mathbf{d}, k}, k=1, \ldots, N_{d}$, where $N_{d}$ is the number of discrete variables to be treated; fast BBM will be applied successively to each of them. By setting $k=1$, the first untreated discrete variable will be processed in the next sub-step.

Substep 6-2: Discretize the $k$ th untreated variable $x_{\mathrm{d}, k}$. Suppose its current value as a continuous variable is $\mathbf{x}^{*} \mathbf{d}, \mathbf{k}$. After fixing the treated discrete variables, two relaxed sub-problems are formulated for those untreated discrete variables:

sub1: $\quad \min f\left(\mathbf{x}_{\mathrm{c}}, x_{\mathrm{d}, k}, \mathbf{x}_{\mathrm{d}, k+}\right)$
s.t. $\left\{\begin{array}{l}\mathbf{h}\left(\mathbf{x}_{\mathrm{c}}, \mathbf{x}_{\mathrm{d}}\right)=0, \mathbf{g}^{\min } \leq \mathbf{g}\left(\mathbf{x}_{\mathrm{c}}, \mathbf{x}_{\mathrm{d}}\right) \leq \mathbf{g}^{\max } \\ \mathbf{x}_{\mathrm{c}} \in\left\{\mathbf{x}_{\mathrm{c}} \mid \mathbf{x}_{\mathrm{c}} \in R, \mathbf{x}_{\mathrm{c}}^{\min } \leq \mathbf{x}_{\mathrm{c}} \leq \mathbf{x}_{\mathrm{c}}^{\max }\right\} \\ x_{\mathrm{d}, k} \leq l_{k}, l_{k}=\mathbf{f l o o r}\left(x_{\mathrm{d}, k}^{*}\right) \\ \mathbf{x}_{\mathrm{d}, k+} \in\left\{x_{\mathrm{d}, i} \mid k<i<N_{\mathrm{d}}, i \in N\right\}\end{array} \quad\right.$ s.t. $\left\{\begin{array}{l}\mathbf{h}\left(\mathbf{x}_{\mathrm{c}}, \mathbf{x}_{\mathrm{d}}\right)=0, \mathbf{g}^{\min } \leq \mathbf{g}\left(\mathbf{x}_{\mathrm{c}}, \mathbf{x}_{\mathrm{d}}, \mathbf{x}_{\mathrm{d}}\right) \leq \mathbf{g}^{\max } \\ \mathbf{x}_{\mathrm{c}} \in\left\{\mathbf{x}_{\mathrm{c}} \mid \mathbf{x}_{\mathrm{c}} \in R, \mathbf{x}_{\mathrm{c}}^{\min } \leq \mathbf{x}_{c} \leq \mathbf{x}_{\mathrm{c}} \max ^{\max }\right\} \\ x_{\mathrm{d}, k} \geq u_{k}, u_{k}=\mathbf{c e i l}\left(x_{\mathrm{d}, k}^{*}\right) \\ \mathbf{x}_{\mathrm{d}, k+} \in\left\{x_{\mathrm{d}, i} \mid k<i<N_{\mathrm{d}}, i \in N\right\}\end{array}\right.$

where $\mathbf{x}_{\mathbf{d}, k^{+}}$represents untreated discrete variables, the functions floor()/ceil()are used to get the maximum/ minimum integer numbers no more/less than the included variable.

Taking $\left(\mathbf{x}^{*} \mathbf{c}, \mathbf{x}^{*} \mathbf{d}\right)$ as the initial value, IPM is used again to solve the two sub-problems in (22). We suppose the obtained objective functions are $f_{\min 1}$ and $f_{\min 2}$ respectively. If $f_{\min 1} \leq$ $f_{\min 2}$, the current discrete variable is frozen as $x_{\mathbf{d}, \mathrm{k}}=l_{k}$; otherwise, set $x_{\mathbf{d}, \mathrm{k}}=u_{k}$. Thus, the current variable is treated and remains fixed in the following sub-steps. Then, use $\min \left\{f_{\min 1}\right.$, $\left.f_{\min 2}\right\}$ as the lower bound of the original objective function and the current solution as initial value of next branching operation with $k=k+1 k=k+1$.

Substep 6-3: Judge if $k>N_{d}$, or all discrete variables have already treated. If yes, go to Step 7; otherwise, go back to Substep 6-3 and continue to determine untreated discrete variables by the fast BBM.

Step 7: Output the final optimal solution to the problem.

Compared with the conventional method, the fast BBM only keeps the minimum branch of the objective function instead of traversing all branches in the worst case. So the calculation burden and the required memory can be considerably reduced and the efficiency greatly improved. Although an optimal solution is not guaranteed in theory, in control practice sub-optimal decision is generally acceptable because the practical requirements of power system and the extensive experience of BBM have been taken into full consideration during the designing procedure. 
Table 2 Parameters of gas consumption curves (p.u)

\begin{tabular}{llll}
\hline Generators & $a$ & $b$ & $c$ \\
\hline G1 G7 & 0.02 & 0.85 & 0.13 \\
G8 G9 & 0.05 & 1.26 & 0.33 \\
G10 G11 & 0.45 & 0.52 & 0.26 \\
\hline
\end{tabular}

\section{Implementation of OPC and its Application Results}

The general structure of the control system and the basic procedure of OPC has been previously described. Here some specific aspects for its implantation will be discussed.

\section{Online Update of the Gas-Consumption Parameters}

In (4), the parameters, namely $a_{i}, b_{i}$ and $c_{i}$, are the key to the accurate calculation of the fuel cost. In the offshore oilplatform power system, each gas turbine generators has different generation efficiency, which changes (generally declines) with the increase of service time. These parameters are initially set according to the efficiency curve provided by the manufacturer. Then during the long-term operation, the generation output and the gas consumption are measured in the field. These field measurements are next processed with a curve-fitting identification procedure to update the parameters on line. Since the online updated curves reflect the change of the actual working condition, the obtained gas consumption is much more precise.

Here the field measurements on June 17 to 23, 2014 are selected to show how the online update works. The relationship of gas consumption versus active power is illustrated in Fig. 5. By the classical least-square based identification method, the parameters of gas consumption curves can be updated on line. They are listed in Table 2, where all the coefficients are per-unit values (Note: the base power is $10 \mathrm{MW}$ and the base gas consumption is $2020 \mathrm{~m}^{3}$ ).

\section{Selection of the Weights in the Objective Function}

The objective function of the optimization problem (15) is a weighted sum of three sub-objectives, namely gas consumption, network loss and voltage deviation, which are per unit or dimensionless quantities. Theoretically, the weights could be arbitrarily selected only if they satisfy the following formula: $w_{\mathrm{c}}+w_{\mathrm{p}}+w_{\mathrm{v}}=1, w_{\mathrm{c}}, w_{\mathrm{p}}$, $w_{\mathrm{v}} \in[0,1]$. However, practical experience shows that their values have a significant effect on the optimization results. Here five different control modes, each with a different setting of weights, are taken as case study:

Fig. 6 System status before OPC

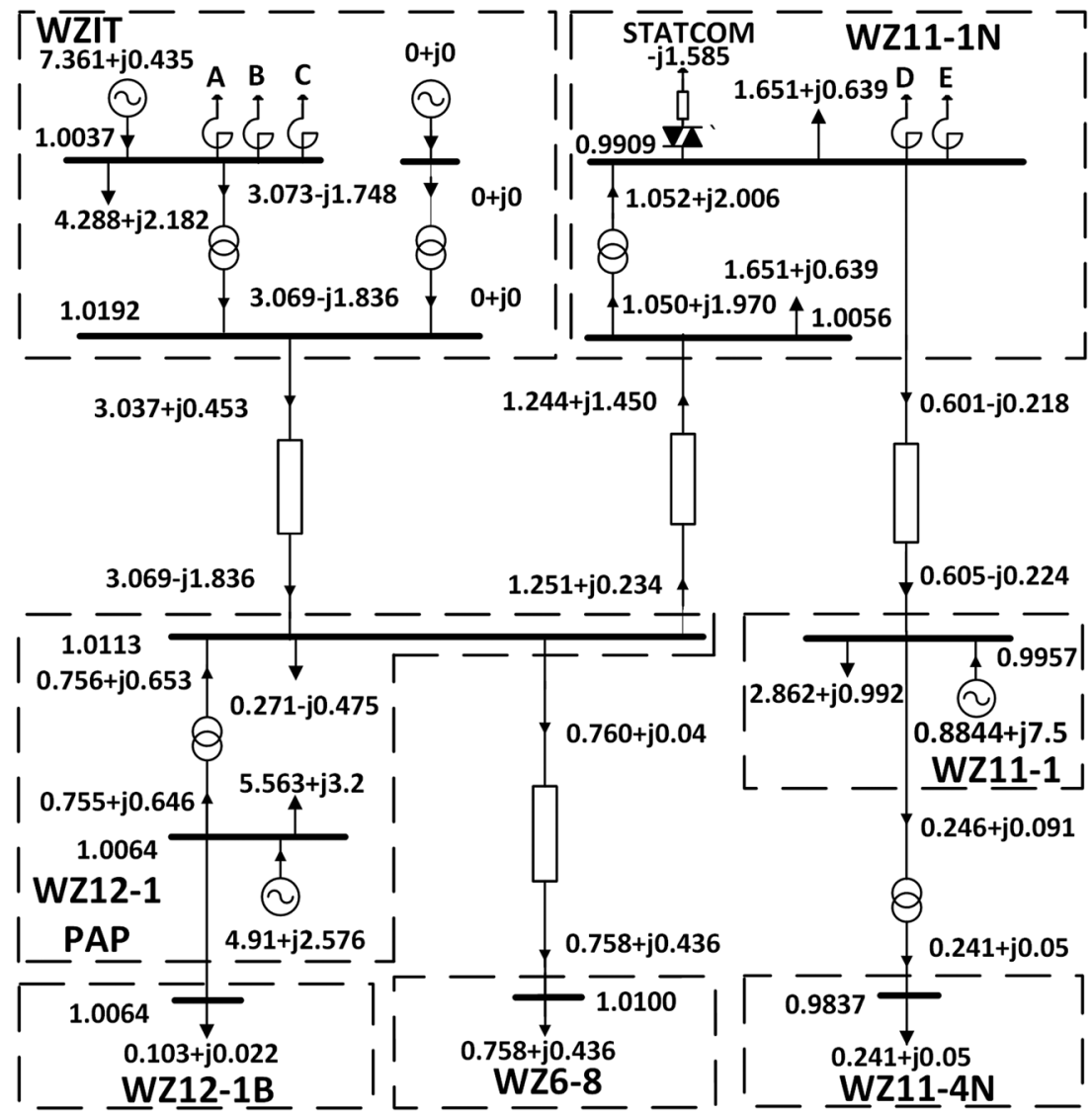


Mode 1: $w_{\mathrm{p}}=1, w_{\mathrm{c}}=w_{\mathrm{v}}=0$, that is, only the network loss is selected as the optimization goal.

Mode 2: $w_{\mathrm{v}}=1, w_{\mathrm{p}}=w_{\mathrm{c}}=0$, that is, only the voltage deviation is selected as the optimization goal.

Mode 3: $w_{\mathrm{c}}=1, w_{\mathrm{p}}=w_{\mathrm{v}}=0$, that is, only the gas consumption is selected as the optimization goal.

Mode 4: $w_{\mathrm{c}}=w_{\mathrm{p}}=0, w_{\mathrm{v}}=0$, that is, the gas consumption and the network loss are equally taken as the optimization goal.

Mode 5: $w_{\mathrm{c}}=0.05, w_{\mathrm{p}}=0.8, w_{\mathrm{v}}=0.15$, that is, all three sub-objectives are taken into account by a composite set of weights, which actually are determined through trial and error.

In order to compare the effects of the above five control modes, we select a particular actual state of WOOPS as the operation scenario before optimization. The corresponding power flow is shown in Fig. 6. By applying the above control modes one by one to this system, we can get the corresponding state of the optimized system, of which the network loss rate, the voltage deviation and the gas consumption are calculated respectively, as shown in Figs. 7, 8, 9.

By comparing the control effects of the five control modes, it is obvious that if a single objective is taken as optimization goal, like Mode 1, 2 and 3, the optimized system will has the best performance in the selected aspect. For instance, Mode 1, 2 and 3 exhibit the best result in minimizing the network loss rate, the voltage deviation and the gas consumption, respectively. But they generally perform poorly in other aspects. Mode 4 incorporates both economic indicators (i.e., network loss and gas consumption), however, without the consideration of the voltage quality. As a result, both network loss and gas consumption are reduced after optimization. But the voltage deviation becomes worse to some extent. Mode 5 accommodate all the three goals. In other words, both economic and security criteria are taken into account. So the three objectives are balanced. Compared to the original state of the system, the network loss rate, the gas consumption and the voltage deviation are all reduced in a coordinated way. Actually, such a compromising control mode is exactly what

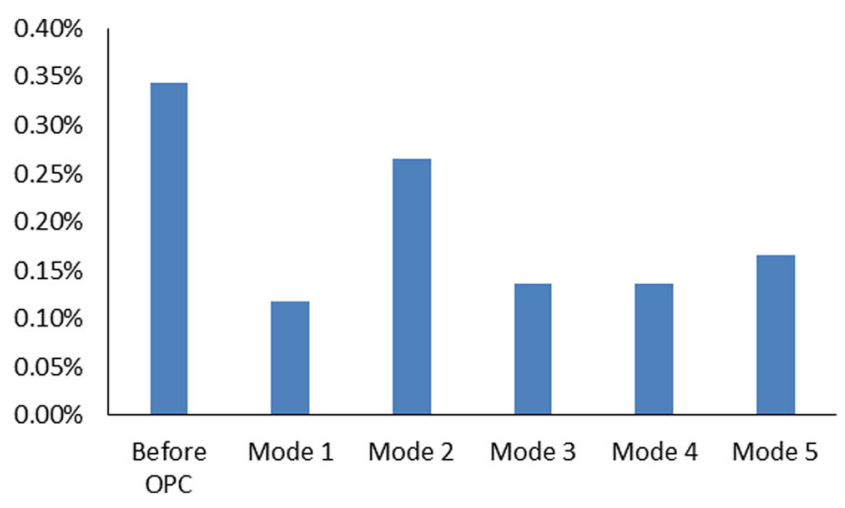

Fig. 7 Power loss rate under different control modes

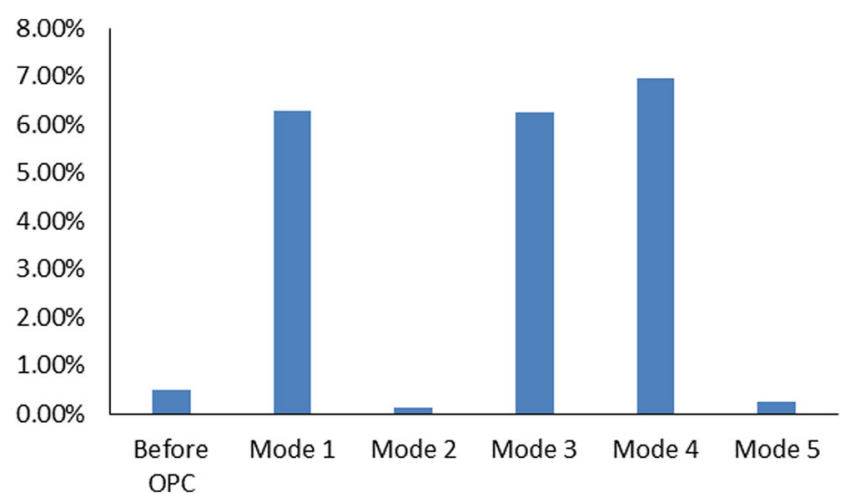

Fig. 8 Voltage deviation under different control modes

the system operators expect. So the weights defined by Mode 5 are adopted in practice.

However, it should be pointed out that the weights of mode 5 depends on the system operating conditions and they should be adjusted when the target system has a major change. According to the experiences with several offshore oilplatform power system, there are some guidelines for selecting the weights of mode 5 :

1) For smaller system, since the voltage fluctuation is relatively larger and the power loss of the networks is not so significant, the weight for voltage should be increased while the weight for the loss should be lowered.

2) The fuel cost is quite different from one system to another, depending on the fuel value, transportation cost of the accompany gas. A higher cost of fuel requires a larger value of $w_{\mathrm{c}}$.

3) In practice, the three weights are initially determined by trial and error. Firstly, $w_{\mathrm{v}}$ is selected to keep the fluctuation of voltage within a limit, for instance $\pm 5 \%$ under typical operating conditions. Then, the ratio of $w_{\mathrm{c}}$ and $w_{\mathrm{p}}$ is set to make the economic value of electricity and fuel comparable. Finally, with some trial and error under different scenarios, the weights are determined. However, they can be adjusted afterward by system operators when more experiences are accumulated in practice.

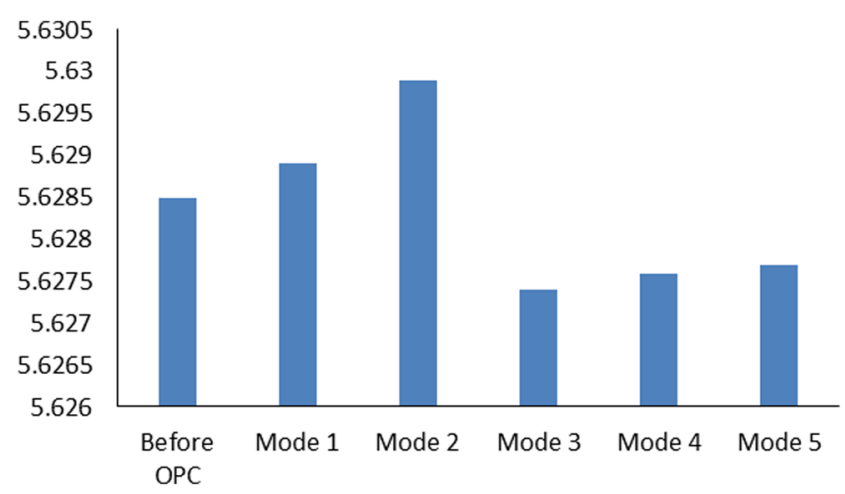

Fig. 9 Gas consumption under different control modes 
Fig. 10 The power flow before and after OPC

\section{Typical Field Test Results}

The developed optimal power control has been implemented as an advanced function in the improved EMS shown in Fig. 3. The EMS operates in a computer system including two backup servers, whose CPU is Intel Xeon E5-2620 V2 $(2.1 \mathrm{GHz}, 7.1 \mathrm{GT} / \mathrm{s})$. The whole control system was put into operation in 2013. The above-mentioned compromising control mode (No.5) was adopted to achieve coordinated optimization of security and efficiency for the oil-platform power system. A typical field test result is provided hereafter. The total load of the system is about $16 \mathrm{MW}$. The power flow before and after the optimization is shown in Fig. 10, where the quantities in the brackets denote the optimized system variables including power, bus voltage, OLTC transformer position and reactor status. Table 3 shows the comparison of system performance before and after the optimal power control.

It can be found that the power output of each generator has been adjusted after the optimal power control with the recommended weights. In particular, the power of G10 on WZIT platform is reduced to 0 from $3.19 \mathrm{MW}$ before optimization, that is, the generator is shut down after the OPC. The power system has its network loss rate lowered from $0.34 \%$ to $0.12 \%$, voltage deviation from $0.39 \%$ to $0.12 \%$ and the total gas consumption from 2.845 p.u. to 2.371 p.u., which fully demonstrates that the OPC has considerably improved the operation performance of the target system.

For this practical case, if the conventional BBM is used, under the worst circumstance there are 23,328 $\left(2^{5} \times 9^{3}\right)$ branches to be traversed. It is a huge work. But the fast BBM only need to solve 8 branch problems at most, each for a discrete variables. Consequently the efficiency of the algorithm is enhanced dramatically. In this specific case, the optimization problem is solved with only 23 iterations, which takes the server about $0.08 \mathrm{~s}$. Therefore, the optimal power control can be executed in real time.

\section{Conclusions}

Secure and economical operation of the offshore oil-platform power system plays a key role in the efficient exploitation of offshore oil and gas. In this paper, an SOA-based multi-objective optimal power control (OPC) is developed as an advanced control function of the EMS. By incorporating network loss,
Table 3 System performance before and after OPC

\begin{tabular}{llll}
\hline & Power Loss Rate (\%) & Voltage Deviation (\%) & Gas Consumption (p.u.) \\
\hline Before OPC & $0.34 \%$ & $0.39 \%$ & 2.845 \\
After OPC & $0.15 \%$ & $0.12 \%$ & 2.371 \\
\hline
\end{tabular}


gas consumption and voltage deviation into a synthesized objective function and taking the various operational constraints into account, the power-control task is formulated into a mixed-integer nonlinear optimized problem. To achieve quasi-real-time decision making, we have implemented an efficient solution to the problem by combining the interior point method and the fast branch and bound method. Particularly, the parameters of the gas consumption are updated online to reflect the current operating performance of gas turbines. The developed optimal power control system has been put into practical use in the Weixinan offshore oilplatform power system (China's first of its kind). Field test results show that it can reach an optimized control strategy in less than $0.1 \mathrm{~s}$ and thus achieve online optimal control of the system. Compared with system status without the OPC, network loss rate, voltage deviation and gas consumption can be reduced by $55 \%, 69 \%$ and $16 \%$, respectively. This has fully demonstrated the effectiveness of the developed OPC system in enhancing power quality as well as operational efficiency of the offshore oil-platform power system, which in turn will improve the efficiency of offshore oil and gas exploitation.

Acknowledgements This work was supported by National Natural Science Foundation of China (Grant No. 51322701) and National High Technology Research and Development Program of China (Grant No. 2012AA050216).

\section{Compliance with Ethical Standards}

Conflict of Interest The authors declare that they have no conflict of interest.

\section{References}

1. U.S. Energy Information Administration (2014) China country energy brief analysis. http://www.eia.gov/countries/analysisbriefs/ China/china.pdf

2. BP (2012) BP Statistical Review of World Energy 2012, http:// www.bp.com/content/dam/bp/pdf/statistical-review/statistical_ review_of_world_energy_2013.pdf

3. Gjolberg O, Johnsen T (1999) Risk management in the oil industry: can information on long-run equilibrium prices be utilized? Energy Econ 21(6):517-527

4. Neffati A, Guemri M, Caux S, Fadel M (2013) Energy management strategies for multi source systems. Electr Power Syst Res 102:4249

5. Miguel P, Neves L, Gomes Martins A (2014) Methodology to simulate the impact of a large deployment of a residential energy management system in the electricity grid. Electr Power Syst Res 116: 399-407

6. Debbarma S, Saikia LC, Sinha N (2013) AGC of a multi-area thermal system under deregulated environment using a noninteger controller. Electr Power Syst Res 95:175-183

7. Calderaro V, Conio G, Galdi V, Piccolo A (2012) Reactive power control for improving voltage profiles: a comparison between two decentralized approaches. Electr Power Syst Res 83(1):247-254
8. Marvik JI, Øyslebø EV, Korpås M (2013) Electrification of offshore petroleum installations with offshore wind integration. Renew Energy 50:558-564

9. Årdal AR, Undeland T, Sharifabadi K (2012) Voltage and frequency control in offshore wind turbines connected to isolated oil platform power systems. Energy Procedia 24:229-236

10. Jifen Z, Peng H, Jun L (2009) Security and stability control system for offshore platforms power grid. Pet Explor Dev 36(2):237-241

11. Sučić S, Dragičević T, Capuder T, Delimar M (2011) Economic dispatch of virtual power plants in an event-driven service-oriented framework using standards-based communications. Electr Power Syst Res 81(12):2108-2119

12. IEC 61970 (2003) Energy Management System Application Prog ram Interface (EMS-API) Part 301 : Common Information Model (CIM)Base

13. McMorran AW, Ault GW, Elders IM, Foote CET, Burt GM, McDonald JR (2004) Translating CIM XML power system data to a proprietary format for system simulation. Power Systems, IEEE Transactions on 19(1):229-235

14. Farrokhabadi M, Vanfretti L (2014) An efficient automated topology processor for state estimation of power transmission networks. Electr Power Syst Res 106:188-202

15. Xie N, Torelli F, Bompard E (2015) Alfredo Vaccaro, a graph theory based methodology for optimal PMUs placement and multiarea power system state estimation. Electr Power Syst Res 119:25-33

16. Zamora-Cárdenas A, Fuerte-Esquivel CR (2011) State estimation of power systems containing facts controllers. Electr Power Syst Res 81(4):995-1002

17. Tebianian H, Jeyasurya B (2015) Dynamic state estimation in power systems: modeling, and challenges. Electr Power Syst Res 121: 109-114

18. Frank S, Steponavice I, Rebennack S (2012) Optimal power flow: a bibliographic survey I. Energy Syst 3(3):221-258

19. Frank S, Steponavice I, Rebennack S (2012) Optimal power flow: a bibliographic survey II. Energy Syst 3(3):259-289

20. Liang R-H, Tsai S-R, Chen Y-T, Tseng W-T (2011) Optimal power flow by a fuzzy based hybrid particle swarm optimization approach. Electr Power Syst Res 81(7):1466-1474

21. Gomez-Gonzalez M, López A, Jurado F (2012) Optimization of distributed generation systems using a new discrete PSO and OPF. Electr Power Syst Res 84(1):174-180

22. Numnonda T, Annakkage UD (1999) Optimal power dispatch in multinode electricity market using genetic algorithm. Electr Power Syst Res 49(3):211-220

23. Moeini-Aghtaie M, Abbaspour A, Hajipour E (2014) A decomposed solution to multiple-energy carriers optimal power flow. Power Systems, IEEE Transactions on 29(2):707-716

24. Estevam CRN, Rider MJ, Amorim E, Mantovani JRS (2010) Reactive power dispatch and planning using a non-linear branchand-bound algorithm. Generation, Transmission \& Distribution, IET 4(8):963-973

25. Bose S, Pal S, Natarajan B, Scoglio CM, Das S, Schulz NN (2012) Analysis of optimal reconfiguration of shipboard power systems. Power Systems, IEEE Transactions on 27(1):189-197

26. Capitanescu F, Wehenkel L (2013) Experiments with the interiorpoint method for solving large scale optimal power flow problems. Electr Power Syst Res 95:276-283

27. Introduction to Ipopt (2015) https://projects.coin-or.org/Ipopt/ browser/stable/3.11/Ipopt/doc/documentation.pdf?format=raw

28. Oliveira EJ, Oliveira LW, Pereira JLR, Honório LM, Silva Junior IC, Marcato ALM (2015) An optimal power flow based on safety barrier interior point method. Int J Electr Power Energy Syst 64: 977-985 